\title{
Conserved Currents and Symmetry Transformations in Local Scattering Theory
}

\author{
K. Kraus \\ Institut für Theoretische Physik der Universität Marburg, Germany \\ L. J. LANDAU \\ Seminar für Theoretische Physik der ETH Zürich, Switzerland
}

Received September 9, 1971

\begin{abstract}
The relation between conserved currents and symmetries of the $S$-matrix is investigated within the framework of Wightman field theory. Assuming a complete particle interpretation with no massless particles, it is shown that every conserved current yields a self-adjoint charge operator which acts additively on $n$-particle states and commutes with the $S$-matrix. For currents satisfying current algebra relations of a group $\mathscr{G}$, the corresponding charges generate a unitary representation of $\mathscr{G}$.
\end{abstract}

\section{Introduction}

The relation between conserved currents and associated symmetry transformations has been studied in much detail [1]. In a local relativistic framework without massless particles it is known that a conserved current always defines a symmetric (hermitian) operator (the "charge") [2], but in order to uniquely define a one-parameter group of unitary operators the charge must be essentially self-adjoint. In addition, even if a set of self-adjoint charges satisfy the commutation relations of a Lie algebra on a dense subset of Hilbert space, it does not necessarily follow that the charges generate a unitary representation of the associated Lie group [3]. As suggested by Orzalesi in [1], the asymptotic states of a scattering theory might provide a domain on which the charges are sufficiently well-behaved to avoid the mathematical difficulties mentioned above. In this note we point out that the locality of the current always leads rigorously to the conclusion that the charge acts additively on the asymptotic multiparticle states, and that these states provide the necessary domain required to show that the charge is essentially self-adjoint. If a set of conserved currents satisfy the commutation relations of a Lie algebra, the charges will satisfy the same commutation relations on the 
asymptotic states and will generate a unitary representation $U(\mathscr{G})$ of the associated Lie group. These unitary operators act multiplicatively on the asymptotic states and commute with the $S$-matrix. In view of the fact that the interacting fields can be defined on a dense set of asymptotic states [4], these states form a natural domain on which to study the local action of $U(\mathscr{G})$ on the interacting fields. The transformation properties of the currents under $U(\mathscr{G})$ are investigated in this way.

The discussion is carried out within the Wightman framework of field theory [5]. Among the irreducible local Poincare covariant fields $\Phi_{\alpha}(x)$ are a set of hermitian conserved currents $j_{i}^{\mu}(x), i=1, \ldots, N ; \mu=0$, $\ldots, 3$, and $\partial_{\mu} j_{i}^{\mu}(x)=0$. The currents are not necessarily four-vectors, the additional tensor indices being included in the index $i$. In addition to the usual Wightman axioms the following assumptions are essential here.

i) The existence of a unique vacuum $|v a c\rangle$ and absence of masszero particles.

ii) Asymptotic completeness with a finite number of particles at each mass value.

Let $D$ denote the domain generated from the vacuum by localized polynomials in the fields: fields averaged with test functions from $\mathscr{D}$ (infinitely differentiable with compact support in coordinate space). As usual [1] a local charge $j^{0}\left(f_{R} f_{T}\right)$ is defined with $f_{R}(\boldsymbol{x}), f_{T}\left(x^{0}\right) \in \mathscr{D}$,

$$
f_{R}(x)=\left\{\begin{array}{lll}
1 & \text { for }|x|<R & f_{T}\left(x^{0}\right)=0 \text { for } \quad\left|x^{0}\right|>T \\
0 & \text { for }|x|>R+\lambda, & \int f_{T}\left(x^{0}\right) d x^{0}=1 .
\end{array}\right.
$$

A charge operator $Q$ is defined on the domain $D$ by

$$
\begin{aligned}
Q|\mathrm{vac}\rangle & =0 \\
Q P|\mathrm{vac}\rangle & =\left[j^{0}\left(f_{R} f_{T}\right), P\right]|\mathrm{vac}\rangle
\end{aligned}
$$

where $P$ is a localized polynomial in the fields, and expression (1.1) is independent of $R$ for $R$ sufficiently large, due to the local commutativity of the currents and fields. Expressions such as (1.1) will always be interpreted as meaning that $R$ is to be chosen "sufficiently large". The charge $Q$ is thus defined on the dense domain $D$ and is symmetric there:

$$
\begin{aligned}
& \left\langle P_{1} \operatorname{vac} \mid Q P_{2} \mathrm{vac}\right\rangle-\left\langle Q P_{1} \mathrm{vac} \mid P_{2} \mathrm{vac}\right\rangle \\
= & \left\langle\operatorname{vac}\left|\left[j^{0}\left(f_{R} f_{T}\right), P_{1}^{*} P_{2}\right]\right| \operatorname{vac}\right\rangle=0 .
\end{aligned}
$$

The first step follows from substituting the definition of the charge (1.1), and the last step follows from the absence of mass-zero particles [6].

It is now essential to extend the definition of $Q$ to the asymptotic multiparticle states. We briefly recall here the Haag-Ruelle construction 
of asymptotic states [7]. Let $|\boldsymbol{p} i\rangle$ be one-particle states of a given mass $M$ with momentum $\boldsymbol{p}$ and type $i$ (the index $i$ representing the spin and any other quantum numbers), and let $C$ be a given compact region of momentum space. Then a set of operators $b_{i}$ can be constructed so that $b_{i}$ creates a one-particle state of mass $M$ from the vacuum and such that the wave function

$$
\left\langle\boldsymbol{p} j\left|b_{i}\right| \mathrm{vac}\right\rangle=\delta_{i j}
$$

in the region $C$. The operators $b_{i}$ are finite sums of the form

$$
b_{i}=\sum_{\alpha} \int d^{4}(x) h_{\alpha}(x) P_{\alpha}(x)
$$

where $P_{\alpha}$ is a polynomial in the fields averaged with test functions from $\mathscr{D}, P_{\alpha}(x)$ is the translate by amount $x$ of $P_{\alpha}$, and $h_{\alpha}(x)$ is a rapidly decreasing test function with support in momentum space confined to a neighborhood of the mass shell $p^{2}=M^{2}$. We further define

$$
b(f, t)=\sum_{i} \int d^{3}(\boldsymbol{x}) \tilde{f}_{i}(\boldsymbol{x}, t) b_{i}(\boldsymbol{x}, t)
$$

with

$$
\tilde{f}_{i}(\boldsymbol{x}, t)=(2 \pi)^{-3} \int d^{3}(\boldsymbol{p}) f_{i}(\boldsymbol{p}) e^{i\left(\boldsymbol{p} \cdot \boldsymbol{x}-\sqrt{\boldsymbol{p}^{2}+M^{2}} t\right)}
$$

and the support of $f_{i}(\boldsymbol{p})$ is contained in the compact region $C$ of momentum space. Then $b(f, t)$ creates a one-particle state with wave function $f_{i}(\boldsymbol{p})$ :

$$
b(f, t)|\operatorname{vac}\rangle=|f\rangle \quad \text { with }\langle\boldsymbol{p} i \mid f\rangle=f_{i}(\boldsymbol{p})
$$

independent of $t$.

The $n$-particle states with compact support wave functions are constructed as the strong limit

$$
\left|f_{1} \ldots f_{n}\right\rangle=\lim _{t \rightarrow \infty} b\left(f_{1}, t\right) \ldots b\left(f_{n}, t\right)|\mathrm{vac}\rangle .
$$

Here the operators $b\left(f_{k}, t\right)$ may belong to different masses $M$. It will now be shown that the charge $Q$ is defined on these asymptotic states and is self-adjoint.

\section{The Self-Adjointness of the Charge}

In Eq. (1.3) replace $P_{\alpha}$ by $P_{\alpha}^{Q} \equiv\left[j^{0}\left(f_{R} f_{T}\right), P_{\alpha}\right]$. In this way we obtain

and

$$
b_{i}^{Q}=\sum_{\alpha} \int d^{4}(x) h_{\alpha}(x) P_{\alpha}^{Q}(x)
$$

$$
b^{Q}(f, t)=\sum_{i} \int d^{3}(\boldsymbol{x}) \tilde{f}_{i}(\boldsymbol{x}, t) b_{i}^{Q}(\boldsymbol{x}, t)
$$


Then from the construction discussed above of one and many-particle states, it is clear that $b^{Q}(f, t)|\mathrm{vac}\rangle$ is a one-particle state of mass $M$, which we denote by $\left|f^{Q}\right\rangle$. Furthermore the strong limit

$$
\lim _{t \rightarrow \infty} b\left(f_{1}, t\right) \ldots b^{Q}\left(f_{i}, t\right) \ldots b\left(f_{n}, t\right)|\mathrm{vac}\rangle
$$

exists and is equal to $\left|f_{1} \ldots f_{i}^{Q} \ldots f_{n}\right\rangle$. This follows from the relative locality of $P_{\alpha}$ and $P_{\alpha}^{Q}$.

Translation invariance of $Q$ implies

$Q b\left(f_{1}, t\right) \ldots b\left(f_{n}, t\right)|\mathrm{vac}\rangle=\sum_{i=1}^{n} b\left(f_{1}, t\right) \ldots b^{Q}\left(f_{i}, t\right) \ldots b\left(f_{n}, t\right)|\mathrm{vac}\rangle$

if we replace the rapidly decreasing test functions $h_{\alpha}$ and $\tilde{f}_{i}$ which occur in the definitions (1.3) and (1.4) by compact support test functions $h_{\alpha}^{\prime}$ and $\tilde{f}_{i}^{\prime}$. If we now let $h_{\alpha}^{\prime}$ and $\tilde{f}_{i}^{\prime}$ converge to $h_{\alpha}$ and $\tilde{f}_{i}$, then because the fields and currents are tempered fields, the vectors occurring in (2.1) will converge strongly. Since $Q$ is densely defined and symmetric, we may without loss of generality assume it is a closed operator, and it may then be concluded that (2.1) holds also for rapidly decreasing test functions.

Finally we note that because $b\left(f_{1}, t\right) \ldots b\left(f_{n}, t\right) \mid$ vac $\rangle$ converges strongly to $\left|f_{1} \ldots f_{n}\right\rangle, \sum_{i} b\left(f_{1}, t\right) \ldots b^{Q}\left(f_{i}, t\right) \ldots b\left(f_{n}, t\right) \mid$ vac $\rangle$ converges strongly to $\sum_{i}\left|f_{1} \ldots f_{i}^{Q} \ldots f_{n}\right\rangle$, and because $Q$ is closed, it follows that $Q$ is defined on $\left|f_{1} \ldots f_{n}\right\rangle$ and maps it into $\sum_{i}\left|f_{1} \ldots f_{i}^{Q} \ldots f_{n}\right\rangle$. Due to this additivity, $Q$ has the same action on the "in" or "out" states. Thus ${ }^{1}$ :

Theorem 2.1. The charge $Q$ is defined on the asymptotic n-particle states $\left|f_{1} \ldots f_{n}\right\rangle$ and acts additively:

$$
Q\left|f_{1} \ldots f_{n}\right\rangle=\sum_{i}\left|f_{1} \ldots f_{i}^{Q} \ldots f_{n}\right\rangle .
$$

$Q$ commutes with the $S$-matrix.

To determine more explicitly the action of $Q$ on the one-particle states, note that the wave function $f_{\tilde{j}}^{Q}(\boldsymbol{p})$ associated with $Q|f\rangle$ is given by

$$
\begin{aligned}
f_{j}^{Q}(\boldsymbol{p}) \equiv\langle\boldsymbol{p} j|Q| f\rangle & =\left\langle\boldsymbol{p} j\left|b^{Q}(f, t)\right| \mathrm{vac}\right\rangle=\sum_{i} \int d^{3}(\boldsymbol{x}) \tilde{f}_{i}(\boldsymbol{x}, t) e^{i p \cdot x}\left\langle\boldsymbol{p} j\left|b_{i}^{Q}\right| \mathrm{vac}\right\rangle \\
& =\sum_{i} f_{i}(\boldsymbol{p})\left\langle\boldsymbol{p} j\left|b_{i}^{Q}\right| \mathrm{vac}\right\rangle=\sum_{i} q_{j i}(\boldsymbol{p}) f_{i}(\boldsymbol{p})
\end{aligned}
$$

where we have defined

$$
q_{j i}(\boldsymbol{p})=\left\langle\boldsymbol{p} j\left|b_{i}^{Q}\right| \mathrm{vac}\right\rangle
$$

${ }^{1}$ This result is obtained by Orzalesi [1] and Lopuszanski [8] where, however, domain questions are not discussed in detail. 
which is an infinitely differentiable function of $\boldsymbol{p}$ because of the locality of $P_{\alpha}^{Q}$ [7]. We use definition (2.2) only for $\boldsymbol{p}$ in the region $C$ where $\left\langle\boldsymbol{p} j\left|b_{i}\right|\right.$ vac $\rangle$ has the form (1.2). Because $Q$ is symmetric, $q_{j i}(\boldsymbol{p})=q_{i j}(\boldsymbol{p})^{*}$.

For any given compact region $C$ we may find $b_{i}$ 's such that (1.2) holds, and the definitions (2.2) must agree for any chosen $b_{i}$ since $Q$ has a unique closure. We thus see that $Q$ is defined on all one-particle states with compact support wave-functions and acts on these states by matrix multiplication with the infinitely differentiable hermitian matrix function $q_{j i}(p)$. It is clear then that the operator $Q$ is bounded on states with a fixed number of asymptotic particles with fixed masses and with momentum space wave-functions whose supports are restricted to a given bounded region. Denoting by $D^{\mathrm{ex}}$ the set of states with a finite number of asymptotic particles with momentum space wave-functions from $\mathscr{D}$, it follows that such states form a dense set of analytic vectors for $Q$ (with an infinite radius of convergence). By Lemma 5.1 of Nelson [3], $Q$ is self-adjoint. Thus:

Theorem 2.2. The charge operator $Q$ is self-adjoint.

Examples of currents with high tensor-character are easily constructed in the free-field case. For example, for a charged scalar field, the current

$$
j_{v_{1} v_{2}}^{\mu}(x)=i / 2\left(: \partial_{v_{1}} \Phi^{*}(x) \overleftrightarrow{\partial^{\mu}} \partial_{v_{2}} \Phi(x):+: \partial_{v_{2}} \Phi^{*}(x) \overleftrightarrow{\partial^{\mu}} \partial_{v_{1}} \Phi(x):\right)
$$

is local, hermitian, and conserved, and leads to charges with tensor character. Such currents are discussed by Kibble [9] and more recently by Lopuszanski [8]. As pointed out in these references, the analogue of such currents are not expected to exist for interacting fields since the associated charges are polynomials in the momenta of the particles, and such polynomials are not expected to be conserved if the particles interact (see also Ref. [10]).

It is possible that the charge associated with a current is identically zero. An example of such a current is provided by the free massive fourvector hermitian field $A^{\mu}(x)$ describing a spin-one particle, for which $\partial_{\mu} A^{\mu}=0$, and for which the charge defined by equation (1.1) is identically zero.

\section{Current Algebra Relations and Group Structure}

We consider now $N$ hermitian conserved currents $j_{i}^{\mu}(x), i=1, \ldots, N$; $\mu=0, \ldots, 3$ which satisfy current algebra commutation relations of the form

$$
\left[j_{i}^{0}\left(f_{R} f_{T}\right), j_{k}^{0}(f)\right]=-i \sum_{m} C_{i k}^{m} j_{m}^{0}(f)+S(\nabla f) \quad \text { on } \quad D
$$


Here $C_{i k}^{m}$ are the structure constants of a simply-connected $N$-parameter Lie group $\mathscr{G}, f$ is an arbitrary test function from $\mathscr{D}$, and $S(\nabla f)$ denotes a possible "Schwinger term" involving a local operator $S(x)$ and the spatial derivatives of $f$.

Theorem 3.1. The one-parameter groups $U_{i}\left(s_{i}\right)=\exp \left(i s_{i} Q_{i}\right)$ generate a continuous unitary representation $U(\mathscr{G})$ of the group $\mathscr{G}$.

Proof. According to Theorem 5 of Nelson [3] it suffices to prove the existence of a common invariant dense domain $D_{0}$ such that

$$
\left[Q_{i}, Q_{k}\right]=-i \sum_{m} C_{i k}^{m} Q_{m} \quad \text { on } \quad D_{0}
$$

and such that

$$
\Delta \equiv Q_{1}^{2}+\cdots+Q_{N}^{2}
$$

is essentially self-adjoint on $D_{0}$. The set $D^{\text {ex }}$ considered above provides a dense set of analytic vectors for $\Delta$, so $\Delta$ is essentially self-adjoint on $D^{\text {ex }}$. It is easy to show from the Jacobi identity that (3.2) holds on the domain $D$ as a consequence of (3.1), and since the charges are self-adjoint, Eq. (3.2) also holds on $D^{\text {ex }}$ : For $\Phi_{1} \in D, \Phi_{2} \in D^{\text {ex }}$

$$
\begin{aligned}
\left\langle\Phi_{1} \mid\left[Q_{i}, Q_{k}\right] \Phi_{2}\right\rangle & =-\left\langle\left[Q_{i}, Q_{k}\right] \Phi_{1} \mid \Phi_{2}\right\rangle=-i\left\langle\sum_{m} C_{i k}^{m} Q_{m} \Phi_{1} \mid \Phi_{2}\right\rangle \\
& =\left\langle\Phi_{1} \mid-i \sum_{m} C_{i k}^{m} Q_{m} \Phi_{2}\right\rangle .
\end{aligned}
$$

Since $D$ is dense, Eq. (3.2) holds on $D^{\text {ex }}$ and the theorem is proved.

If no Schwinger term is present in (3.1) then the currents transform according to the adjoint representation of $\mathscr{G}$ on the dense domain $\hat{D}^{\text {ex }}$ consisting of states from $D^{\text {ex }}$ with non-overlapping momentum-space wave functions [4], i.e. wave functions which are pairwise disjoint in velocity space $\boldsymbol{p} / \sqrt{\boldsymbol{p}^{2}+M^{2}}$. To show this first observe that the interacting fields are defined on $\hat{D}^{\text {ex }}$ (Sect. 6 of [4]) and in particular the currents are defined there. The explicit form of $Q_{i}$ derived above implies the invariance of $\hat{D}^{\text {ex }}$ under $Q_{i}$ and $U_{i}(s)=\exp \left(i s Q_{i}\right)$. In addition the commutation relation

holds on $\hat{D}^{\text {ex }}$ :

$$
\left[Q_{i}, j_{k}^{0}(f)\right]=-i \sum_{m} C_{i k}^{m} j_{m}^{0}(f)
$$

Let $\Phi_{1} \in \hat{D}^{\mathrm{ex}}$ and $\Phi_{2} \in D$. Then

i.e.

$$
\left\langle\Phi_{1}\left|\left[Q_{i}, j_{k}^{0}\left(f^{*}\right)\right]\right| \Phi_{2}\right\rangle=\left\langle\Phi_{1}\left|-i \sum_{m} C_{i k}^{m} j_{m}^{0}\left(f^{*}\right)\right| \Phi_{2}\right\rangle,
$$

$$
\left\langle j_{k}^{0}(f) \Phi_{1}\left|Q_{i}\right| \Phi_{2}\right\rangle=\left\langle j_{k}^{0}(f) Q_{i} \Phi_{1}-i \sum_{m} C_{i k}^{m} j_{m}^{0}(f) \Phi_{1} \mid \Phi_{2}\right\rangle .
$$


Now $Q_{i}$ is obtained by closure from the localized states $D$ and it may therefore be concluded that (3.4) holds for all $\Phi_{2}$ in the domain of $Q_{i}$. Since $Q_{i}$ is self-adjoint this implies that $j_{k}^{0}(f) \Phi_{1}$ is in the domain of $Q_{i}$ and that

$$
Q_{i} j_{k}^{0}(f) \Phi_{1}=j_{k}^{0}(f) Q_{i} \Phi_{1}-i \sum_{m} C_{i k}^{m} j_{m}^{0}(f) \Phi_{1} .
$$

Therefore Eq. (3.3) holds on $\hat{D}^{\mathrm{ex}}$.

Consider now the matrix element

$$
\begin{gathered}
\left\langle\Phi_{1}\left|U_{i}(s) j_{k}^{0}(f)\right| \Phi_{2}\right\rangle \quad \Phi_{1}, \Phi_{2} \in \hat{D}^{\mathrm{ex}} \\
=\left\langle U_{i}(s)^{-1} \Phi_{1}\left|j_{k}^{0}(f)\right| \Phi_{2}\right\rangle=\sum_{n=0}^{\infty}(i s)^{n} / n !\left\langle Q_{i}^{n} \Phi_{1}\left|j_{k}^{0}(f)\right| \Phi_{2}\right\rangle
\end{gathered}
$$

where $U_{i}(s)^{-1} \Phi_{1}$ has been expanded into a convergent power series since $\Phi_{1}$ is an analytic vector for $Q_{i}$. Successive application of the commutation relation (3.3) leads to

$$
\left\langle\Phi_{1}\left|U_{i}(s) j_{k}^{0}(f)\right| \Phi_{2}\right\rangle=\sum_{n=0}^{\infty} \sum_{m=1}^{N}\left\langle j_{m}^{0}\left(f^{*}\right) \Phi_{1}\left|\left[\left(e^{i s \alpha_{i}}\right)_{m k} e^{i s Q_{2}}\right]_{n}\right| \Phi_{2}\right\rangle
$$

where []$_{n}$ denotes the $n$th term in a power series expansion in $s$ of the quantity in brackets, and $\alpha_{i}$ is the matrix

$$
\left(\alpha_{i}\right)_{m k}=-i C_{i k}^{m}
$$

which is the matrix representation of the Lie algebra of $\mathscr{G}$ in the adjoint representation. Since $\Phi_{2}$ is an analytic vector for $Q_{i}$, the power series in (3.5) converges to

$$
\sum_{m}\left\langle j_{m}^{0}\left(f^{*}\right) \Phi_{1}\left|\left(e^{i s \alpha_{2}}\right)_{m k} U_{i}(s)\right| \Phi_{2}\right\rangle .
$$

Thus on $\hat{D}^{\text {ex }}$

or

$$
U_{i}(s) j_{k}^{0}(f)=\sum_{m} j_{m}^{0}(f)\left(e^{i s \alpha_{i}}\right)_{m k} U_{i}(s)
$$

$$
U_{i}(s) j_{k}^{0}(f) U_{i}(s)^{-1}=\sum_{m} j_{m}^{0}(f)\left(e^{i s \alpha_{i}}\right)_{m k} .
$$

Since exponentials of the form $\exp \left(i s \alpha_{i}\right)$ generate the adjoint representation of $\mathscr{G}$, the following theorem holds.

Theorem 3.2. If the commutation relations (3.1) hold with no Schwinger term, then

$$
U(g) j_{k}^{0}(f) U(g)^{-1}=\sum_{m} j_{m}^{0}(f) A_{m k}(g) \quad \text { on } \quad \hat{D}^{\mathrm{ex}},
$$

where $A_{m k}(g)$ is the adjoint representation of $\mathscr{G}$. 
Although (3.6) has been shown only for the time-component of $j_{k}(f)$, the equation actually holds for all components. This follows from the discussion of the next section, which shows that those charges which are not Lorentz scalars generate a central subgroup, whereas the scalar charges generate an invariant subgroup of $\mathscr{G}$. From this fact it follows by a straightforward generalization of relation (3.3) that the transformation property (3.6) holds for all components of the currents.

\section{The General Form of the Group $\mathscr{G}$}

We suppose that the currents $j_{i}$ are linearly independent: $\sum_{i} a_{i} j_{i}^{0}(f)=0$ for all $f$ implies $a_{i}=0$. However, as the charge $Q$ associated with a current $j=\sum_{i} a_{i} j_{i}$ may vanish, the Lie algebra $\mathscr{Q}$ generated by the charges as given in (3.2) is not necessarily identical with the Lie algebra $\mathscr{J}$ of the currents as determined by the structure constants in (3.1). It is easily seen that if the charge $Q$ vanishes, then the corresponding current $j$ lies in the center of the Lie algebra $\mathscr{J}$. The Lie algebra $\mathscr{Q}$ is therefore a factor algebra of $\mathscr{J}$ with respect to a central subalgebra, and it is the algebra $\mathscr{Z}$ (which is now generated by linearly independent charges) which should be associated with the group $\mathscr{G}$.

Concerning the general form of $\mathscr{G}$, note that because the $Q_{i}$ are additive and linearly independent and are represented on one-particle states of mass $M$ by the matrix functions $q_{i}(M, p)_{m n}$, there exist a finite set $I$ of masses $M$ and a finite set $S$ of $\boldsymbol{p}$-values for which the matrices $q_{i}$ become linearly independent: $\sum_{i} a_{i} q_{i}(M, \boldsymbol{p})=0$ for all $M$ in $I$ and all $\boldsymbol{p}$ in $S$ implies $a_{i}=0$. Thus the Lie algebra 2 has a faithful finite-dimensional representation $r$ by hermitian matrices and thus a positive definite invariant quadratic form exists - with coefficients given by the trace $\operatorname{Tr}\left(r\left(Q_{i}\right) r\left(Q_{j}\right)\right)$. Thus 2 is a compact Lie algebra in the sense of Pontrjagin [11]. It is therefore a direct sum

$$
\mathscr{Q}=\mathscr{C} \oplus \mathscr{R}
$$

where $\mathscr{C}$ is the center of $\mathscr{Q}$, and $\mathscr{R}$ is a compact semisimple ideal. The Lie group of automorphisms of $\mathscr{R}$ is compact, and its Lie algebra is isomorphic to $\mathscr{R}[11]$.

Now the unitary representation of the Lorentz group generates continuous automorphisms of $\mathscr{2}$. It is easily seen that an automorphism of $\mathscr{Q}$ must map the center $\mathscr{C}$ onto itself and the first derived algebra (which in this case is $\mathscr{R}$ ) onto itself. But it then follows that Lorentz 
transformations commute with all elements of $\mathscr{R}$, since the only representation of the Lorentz group in the compact group of automorphisms of $\mathscr{R}$ is the trivial representation. Thus any charge which is not a Lorentz scalar is contained in the center $\mathscr{C}^{2}$.

The natural group of symmetries of the physical system under consideration is not the group $U(\mathscr{G})$ generated by the charges, but its strong closure $\overline{U(\mathscr{G})}$. All elements of $\overline{U(\mathscr{G})}$ will commute with the $S$ matrix because $U(\mathscr{G})$ does, but $\overline{U(\mathscr{G})}$ is not necessarily generated by local currents. The subgroup of "internal" symmetries $U^{\prime}(\mathscr{G})$ consisting of those elements from $U(\mathscr{G})$ which commute with Poincaré transformations, is not necessarily compact, but its closure $\overline{U^{\prime}(\mathscr{G})}$ will be compact. This is illustrated by the example of two complex free fields $\Phi_{1}, \Phi_{2}$ of mass $M$ and the conserved current

$$
j^{\mu}(x)=i e_{1}: \Phi_{1}^{*}(x) \overleftrightarrow{\partial^{\mu}} \Phi_{1}(x):+i e_{2}: \Phi_{2}^{*}(x) \overleftrightarrow{\partial^{\mu}} \Phi_{2}(x):
$$

The charge associated with this current generates a one-parameter group of transformations which is not compact if $e_{1} / e_{2}$ is irrational ${ }^{3}$. The strong closure of this group leads to the compact group $U(1) \otimes U(1)$.

Acknowledgements. We thank R. Haag for his kind hospitality, and him and the other members of the II. Institut für Theoretische Physik, Hamburg, for helpful remarks. One of us (L.J.L.) wishes to thank R. Jost and the other members of the Seminar für Theoretische Physik, Zürich, for several very useful discussions, and J.T. Lopuszanski for an interesting conversation.

\section{References}

1. For a recent review of this work see Orzalesi, C. A.: Rev. Mod. Phys. 42, 381 (1970).

2. See the review [1] or Robinson, D. W.: Symmetries, broken symmetries, currents and charges. In: Symmetry principles and fundamental particles. B. Kursunoglu and A. Perlmutter, Eds. San Francisco: W. H. Freeman and Company 1967.

3. Nelson, E.: Ann. Math. 70, 572 (1959);

Doebner, H. D., Melsheimer, O.: Nuovo Cimento 49 A, 73 (1967).

4. Hepp, K.: On the connection between Wightman and LSZ quantum field theory. In: Axiomatic field theory, M. Chrétien and S. Deser, Eds. New York: Gordon and Breach 1966.

5. Streater, R. F., Wightman, A. S.: PCT, spin \& statistics, and all that. New York: W. A. Benjamin, Inc., 1964.

6. Swieca, J. A.: In, Cargése lectures in physics, Vol. 4, D. Kastler, Ed. New York: Gordon and Breach 1970, and Refs. [1] and [2].

7. Araki,H., Haag, R.: Commun. math. Phys. 4, 77 (1967) and Hepp, K.: Ref. [4].

\footnotetext{
${ }^{2}$ A similar discussion can be found in [10].

${ }^{3}$ Compare Ref. [11], Example 36.
}

18 Commun. math Phys., Vol. 24 
8. Lopuszanski,J.T.: On some properties of physical symmetries, Stony Brook preprint.

9. Kibble, T.W.B.: J. Math. Phys. 6, 1022 (1965).

10. Coleman, S., Mandula, J.: Phys. Rev. 159, 1251 (1967).

11. Pontrjagin, L.: Topological groups, Chap. 11. Princeton: Princeton University Press 1946.

K. Kraus

Physikalisches Institut der Universität

D-87 Würzburg, Germany

Röntgenring 8

(present address)

\section{J. Landau}

Seminar für Theoretische Physik der ETH CH-8049 Zürich, Switzerland

Hönggerberg 